\title{
Physical Activity Level and Factors Affecting Exercise Participation among Nigerian Adults with and Without Diabetes
}

\author{
Authors: \\ Ezema Charles Ikechukwu, ${ }^{1}$ *Mgbeojedo Ukamaka Gloria, ${ }^{1}$ Uchenwoke \\ Chigozie Ikenna, ${ }^{1}$ Ugwueze Vitalis Chinonso, ${ }^{2}$ Uduonu Ekezie \\ Mmanwanne, ${ }^{1}$ Okezue Obinna Chinedu, ${ }^{1}$ Anyachukwu Canice \\ Chukwudi, ${ }^{1}$ John Jeneviv Nene, ${ }^{1}$ Obiekwe Chinwe, ${ }^{2}$ Amarachukwu \\ Charity Nkechi \\ 1. Department of Medical Rehabilitation, University of Nigeria, Enugu Campus, \\ Enugu, Nigeria \\ 2. Department of Physiotherapy, University of Nigeria Teaching Hospital, \\ Ituku-Ozalla, Enugu, Nigeria \\ *Correspondence to amakamgbeojedo@yahoo.com \\ Disclosure: $\quad$ The authors have declared no conflicts of interest. \\ Received: \\ 12.08.20 \\ Accepted: \\ 22.12 .20 \\ Keywords: \\ Exercise participation, diabetes, physical activity level. \\ Citation: \\ EMJ. 2021;6[1]:xx-xx.
}

\begin{abstract}
Background: Diabetes presents a multifaceted challenge to health systems in Nigeria and beyond. Physical activity is a cornerstone of diabetes management but is often underutilised. Despite the positive effects of physical activity on different dimensions of health to patients with diabetes, most fail to maintain long-term adherence to physical activity programmes.
\end{abstract}

Objectives: This study aimed to determine the physical activity level and factors affecting exercise participation among patients with and without diabetes.

Methods: This was a cross-sectional study involving 400 participants recruited by convenience sampling. International Physical Activity Questionnaire (IPAQ) and Exercise Benefit and Barrier Scale (EBBS) questionnaires were used to measure physical activity and perceived benefits and barriers to exercise, respectively. The data collected were analysed using descriptive statistics of percentages and frequency, mean and standard deviation, and independent t-test. The level of significance was set at $\mathrm{p}<0.05$.

Results: The majority of the patients with diabetes (71\%) had low physical activity levels while $52 \%$ of the nondiabetic group were moderately active. There was a significant difference between physical activity levels of patients with diabetes and the nondiabetic group $(p<0.05)$. Physical exertion was reported by both patients with and without diabetes as the greatest barrier to exercise participation.

Conclusion: Patients with diabetes in Nigeria have a low level of physical activity and are also faced with certain barriers which limit their participation in exercise programmes. Exercise barrier identification and public awareness on the health benefits of exercise and physical activity in the prevention and management of diabetes should thus be encouraged. 


\section{INTRODUCTION}

Noncommunicable diseases (NCD) account for approximately $71 \%$ of all deaths globally. Cardiovascular diseases, cancers, chronic respiratory diseases, and diabetes are the four most important $\mathrm{NCD}^{2}$ and contributors to the burden of disease. ${ }^{3}$ The prevalence of these diseases is increasing worldwide, especially in Sub-Saharan Africa (SSA).,4 The risk of death from an NCD is increased by unhealthy lifestyles and behaviours such as sedentary lifestyles, physical inactivity, unhealthy diets, ${ }^{1}$ harmful alcohol and tobacco use, and high blood pressure and cholesterol. ${ }^{3}$ Diabetes is a metabolic disorder of chronic hyperglycaemia, characterised by disturbances to carbohydrate, protein, and fat metabolism resulting from defects in the action of insulin on the target tissues. ${ }^{6,7}$ The International Diabetes Federation (IDF) report estimated that 463 million adults are currently living with diabetes globally, and more than 19 million adults are in the African region; there are approximately 2.7 million adults with diabetes in Nigeria, which is estimated to increase to 47 million by 2045 . Sixty percent of adults living with diabetes are unaware they have the condition and are therefore at high risk of developing serious diabetes-related complications. Approximately $11.3 \%$ of deaths are caused by diabetes, and $87 \%$ of all diabetes-related deaths happen in low- and middle-income countries, with an annual global expenditure of $\$ 760$ billion USD, ${ }^{8}$ and about $\$ 67.03$ billion USD in SSA. ${ }^{9}$

Globally, pooled data from 751 population-based studies reported an increased number of adults with diabetes from 108 million (males: 4.3\%; females: $5.0 \%$ ) in 1980 to 422 million (males: 9.0\%; females: 7.9\%) in 2014.10,11 Substantial evidence exists on the prevalence of diabetes in SSA regions; the prevalence in 2014 was $7.1 \%$, a $129 \%$ increase since $1980 . .^{10,12}$ This increase is unbalanced compared with high-income regions, is affecting younger people, and has enormous economic challenges. ${ }^{13}$ Reports from much older literatures recorded a 2.2\% prevalence rate in Nigeria, ${ }^{14} 4 \%$ in Kenya, ${ }^{15}$ and $0.7 \%$ in Tanzania. ${ }^{16}$ In a systematic analysis report, the overall pooled prevalence of diabetes in the six geo-political zones in Nigeria was 3.0\% in the North-West, $5.9 \%$ in the North-East, 3.8\% in the North-Central zone, $5.5 \%$ in the South-West,
$4.6 \%$ in South-East, and $9.8 \%$ in the South zone. ${ }^{17}$ However, presently, while Nigeria reported a 3\% prevalence rate, Ghana, Kenya, Tanzania, Zambia, the Democratic Republic of Congo, Gambia, and South Africa have reported rates of $1.8 \%, 2.2 \%$, $3.7 \%, 3.4 \%, 6.3 \%, 2.0 \%$, and $9.3 \%$, respectively. 8.18 These observed regional differences could be caused by differences in lifestyle modifications, dietary habits, physical activity, better-resourced health systems, adiposity, genetic factors, ${ }^{19,20}$ limited knowledge, attitude, and practice among community and policy makers in Africa. A study conducted in Nigeria comparing 40 patients with diabetes and 36 nondiabetic controls reported an insulin resistance prevalence of $87.5 \%$ in the diabetics and $27.8 \%$ in the controls. ${ }^{21}$

The increasing burden of diabetes has become a global epidemic, imposing an important economic burden on the already existing resource-limited health systems in SSA. ${ }^{22}$ Awareness level in many SSA nations is low, though is higher among urban residents, ${ }^{23}$ especially in a country like Nigeria that is faced with poor healthcare systems, superstition, poverty, low levels of education, and ignorance. ${ }^{24}$ Some of the adults living with diabetes reside mostly in rural communities where many have superstitious beliefs about most NCD including diabetes. ${ }^{25}$ The high economic burden and complications associated with diabetes can be mitigated if patients have appropriate awareness, knowledge, management, ${ }^{26}$ and prompt diagnosis of the disease.

Lifestyle interventions or modifications, such as avoidance of sedentary lifestyles; regular physical activity; ${ }^{27,28}$ reduction of unhealthy nutrition, especially diets high in sodium and calories and low in dietary fibre; moderate alcohol intake; and tobacco cessation, are important lifestyle recommendations for Type 2 diabetes mellitus management and are relevant in developing nations like Nigeria for cost effectiveness. Regular physical activity reduces high blood sugar level in people with diabetes by improving the sensitivity of skeletal muscles to insulin. According to the World Health Organization (WHO), 60-85\% of people in both developed and developing countries lead sedentary lifestyles, and poor physical activity is the cause of $6 \%$ of global mortality. ${ }^{29}$ The American Diabetes Association (ADA) recommends that each patient with diabetes should do at least 150 minutes of moderate-intensity aerobic physical activity 
and at least 60 minutes of vigorous-intensity physical activity per week. ${ }^{30}$ Barriers to physical activity are personal, social, environmental, and economic and include laziness, lack of stamina or willpower, discouragement from friends and family, fear of injury, embarrassment, weather, cost, age, sex, socio-psychological factors, time, inaccessible or inadequate facilities, transportation, distance, uneven or inappropriate surfaces, and unsupportive peers, amongst others. ${ }^{13,27,31,32} \quad$ Understanding what hinders physical activity for people living with diabetes is important for planning and implementing effective interventions to encourage participation in this population. ${ }^{31}$ 'Diabetes Action Now', a collective project of the WHO and IDF, aims to stimulate and support the adoption of effective measures for surveillance, prevention, and control of diabetes, as well as to achieve a substantial increase in global awareness about diabetes and its complications. ${ }^{33}$

The WHO recommends prevention strategies such as imposing taxes on sugar-sweetened beverages, detailed labelling on food packaging, and the development of education and awareness campaigns to promote physical activity in the community. ${ }^{8}$ Other measures recommended include implementing transportation policies that make it safer for people to walk and ride bicycles; legislating tobacco-free public buildings and spaces; building accessible parks, playgrounds, and community centres; and encouraging physical activity programmes in schools, communities, and health services. ${ }^{29}$ However, this seems to be a far-fetched achievement in a country like Nigeria, where implementation of adopted policies and plans of action are not sustainable. The exercise milieu domain of the EBBS questionnaire is considered a major barrier to exercise participation from the assertion that there are few places structured for exercise and the available few are far away and are unaffordable. The interventions that are effective in one place may not be successful in another, so policies and prevention programmes must be specifically designed for each nation. There is an operational strategy to reduce physical inactivity and diabetes in Nigeria. ${ }^{35}$ However, these are not readily available, accessible, or cost-effective. Owing to this, there is a need to fully address these barriers for effective implementation and optimal management and to invariably reduce the burden of diabetes through physical activity. Earlier studies in Nigeria on barriers to physical activity have been carried out in several patient groups such as stroke survivors, ${ }^{35}$ but to the best of the researcher's knowledge, there is paucity of literature or evidence about the barriers to physical activity among patients with diabetes in Nigeria, hence the following research question: what are the barriers to physical activity participation among patients with diabetes in Nigeria? The authors hypothesised that there would be no significant difference between the physical activity level of those with and without diabetes.

\section{METHODS}

\section{Study Design and Population}

This was a cross-sectional study involving 400 participants (males: 243; females: 157) aged 25 years and above who were conveniently recruited from the University of Nigeria Teaching Hospital, Ituku-Ozalla, Nigeria. The hospital has an outpatient diabetes clinic once a week, as well as inpatient facilities where medical care is provided throughout the week. This outpatient clinic and the hospital was the setting for this study. The sample size was derived on the estimation that the number utilised in this study would be a representative of the total number of all patients with diabetes visiting the clinic monthly. However, this study's participants were only individuals visiting the clinic at the time of data collection and were not representative of all patients with diabetes in the area; the participants were consecutively recruited. The majority of participants were civil servants, middle-class, had at least a senior secondary education, were of Christian faith, seldomly travelled, and lived in bungalows. The patients with diabetes were on antidiabetic medication, though many were noncompliant.

The inclusion criteria included patients who had diabetes, were attending the outpatient diabetes clinic, and did not have other chronic conditions that could affect outcomes. Their medical status was obtained from their outpatient medical cards. Exclusion criteria included pregnant women and individuals with impaired cognitive functioning. 


\section{Sample Size}

All consenting patients seen at the time of data collection participated in this study. The sample size was calculated thus: $n=z^{2} p q / L^{2}$; where $z=1.96$ for $95 \%$ confidence intervals, $L=5 \%$ allowable error, and $p=21.3 \%$ ( $q=100-p$ ). Recruitment continued until the intended number was reached.

\section{Procedure for Data Collection}

Ethical approval was obtained prior to the commencement of the study from the ethical review committee of the University of Nigeria Teaching Hospital. Permission was obtained from the clinic's consultant. An informed consent form was signed by the participants after the study procedures had been thoroughly explained to them. Participation in this study was voluntary. Information on sociodemographic parameters (age, sex, marital status, educational level, and occupational status) was obtained. The questionnaires were researcher administered.

\section{Research Instruments}

\section{International Physical Activity Questionnaire}

Physical activity was measured using the International Physical Activity Questionnaire (IPAQ), which assesses physical activity undertaken across a comprehensive set of domains including leisure physical activity, domestic and gardening activities, work-related physical activity, and transport-related physical activity. ${ }^{36}$ The items were structured to provide separate domain-specific scores for walking, moderate-intensity, and vigorous-intensity activity within each of the domains of work, transportation, domestic chores, gardening, and leisure time. Each activity was also weighted by its relative metabolic cost, referred to as a metabolic equivalent (MET). An average MET score was derived for each type of activity, with MET-minutes per week as the final unit of expression. According to the American College of Sports Medicine (ACSM) guidelines, ${ }^{37}$ one MET represents the energy expenditure for an individual at rest, whereas a 10-MET activity requires 10 -times the resting energy expenditure. For example, all types of walking were included and an average MET value for walking was created. The same procedure was undertaken for moderate-intensity activities and vigorousintensity activities. Physical activity level classification was based on MET-minutes/week in three categories: high, if 7 or more days of any combination of walking or moderate or vigorous intensity activities that achieved at least 3,000 MET-minutes per week was achieved; moderate, if 5 or more days consisted of any combination of walking or moderate or vigorous intensity activities of at least 600 MET-minutes per week; and low, if a patient did not meet any of the aforementioned criteria.

\section{Exercise Benefit and Barrier Scale questionnaire}

Perceived barriers to exercise participation were assessed using the 'Barriers' component of the Exercise Benefit and Barrier Scale (EBBS) questionnaire. $^{38}$ The barrier component utilised had 14 barrier items categorised into four subscales: exercise milieu, time expenditure, physical exertion, and family discouragement.

\section{Data Analysis}

Obtained data was analysed using SPSS Version 21 (IBM, Armonk, New York, USA). Categorical descriptive variables (physical activity levels and sociodemographic profile) were analysed using descriptive statistics of percentage and frequency. Barriers to exercise participation between patients with and without diabetes were analysed in mean and standard deviation. Physical activity (in MET) between those with and without diabetes was analysed using an independent t-test. The alpha level was set at $<0.05$.

\section{RESULTS}

Forty-four percent of the patients with diabetes were adults aged 65 years and above. Most of the participants were male, married, civil servants, and educated to university degree level (Table 1). A total of $71 \%$ of patients with diabetes reported low physical activity levels, while $52 \%$ of the nondiabetic participants reported moderate levels of physical activity (Table 2). Physical exertion and time expenditure subscales of the barriers to exercise participation among diabetic patients had the highest and lowest mean and standard deviation values of $2.90 \pm 0.85$ and $2.66 \pm 0.94$, respectively. The total mean-deviation 
of the exercise participation barrier was $2.78 \pm 0.88$. Physical exertion and family discouragement subscales of the barriers to exercise participation among nondiabetic participants had the highest and lowest mean and standard deviation values of $2.82 \pm 1.63$ and $2.68 \pm 0.80$, respectively. The total mean-deviation of the exercise participation barrier was $2.75 \pm 0.83$ (Table 3).

\section{DISCUSSION}

The majority of participants with diabetes in this study (71.0\%) had low levels of physical activity. This may likely be because of inadequate awareness or detailed education on the benefits of physical activity in diabetes management. This result is much higher than the $31 \%$ from a previous Nigerian study conducted in the South-Western region. ${ }^{39}$ Other SSA studies have reported $39 \%$ for a Rwandan population, ${ }^{40} 25.1 \%$ for a Ghanaian population, and $54.7 \%$ for a Batswana population. ${ }^{41,42}$ Fifty-two percent of Nepalian patients with diabetes, ${ }^{27} 47 \%$ in Malaysia, ${ }^{43}$ and $86 \%$ in Sri-Lanka ${ }^{44}$ were mostly moderately physically active. Regular exercise improves body sensitivity to insulin and helps manage blood glucose levels. The ADA recommends that each patient with diabetes should do at least 150 minutes of moderate-intensity aerobic physical activity weekly. ${ }^{30}$ This suggests that patients living with diabetes in this study were not within this acceptable level. However, a study involving participants from North Carolina, USA revealed that $56 \%$ of patients with diabetes reported at least 150 minutes of moderate or vigorous physical activity weekly. ${ }^{45}$ Population size, instruments, weather, and study designs could be attributable to these differences.

Fifty-two percent of the nondiabetic participants in this study were moderately active. Similar to this finding, $58 \%$ of adults without diabetes in a USA study were physically active (moderate or vigorous activity). ${ }^{46}$ Individuals with chronic diseases in general and diabetes in particular usually stay away from physical activity for fear of worsening their condition or triggering a hypoglycaemic crisis. ${ }^{47}$ There was a statistically significant difference between physical activity levels using MET of patients with diabetes and nondiabetic participants. The result revealed that the patients with diabetes had a mean value of 563.73 while nondiabetic participants had a mean value 1,009.470. The mean scoring was obtained from the IPAQ: low (0-599 MET-min/week), moderate (600-2,999 MET-min/week), and high (over 3,000 MET-min/week). ${ }^{48}$ It was surprising that the nondiabetic participants in this study were moderately physically active, as they were presumed to be apparently healthy and as such were expected to be highly active. To explain this finding, it could be that many health workers live sedentary life by virtue of the nature of their jobs, lack of time to get involved in physical activities, and an over-reliance on motorised transport to commute to work instead of walking. The lower levels of physical activity in both groups may be attributed to other factors besides failing health status; some potential barriers to exercise participation include lack of awareness about benefits; lack of national health, educational, and related policies; lack of valuing sport in society; prevailing local cultures; economic and other competing pressures; time constraints; personal motivation; lack of support from family and friends; lack of access to sport facilities; past experiences; and the lack of availability of local physical programmes. ${ }^{49,50}$

The results of this study on the barriers to exercise participation between participants with and without diabetes showed that both groups perceived physical exertion as the strongest barrier to exercise participation. Tiredness and fatigue have been previously reported as important factors that militate against exercise participation. ${ }^{51}$ Evidence of findings from other SSA regions on barriers to physical activity among patients with diabetes are well researched in the literature. Lack of exercising space and no one to exercise with were the most reported barriers to physical activity in Botswana. ${ }^{42}$ In Rwanda, poor health status, lack of motivation, and lack of awareness about the importance of physical activity were the common barriers to physical activity participation. ${ }^{40}$ Other previous studies reported that most of the patients with diabetes were physically inactive because of lack of time and energy, and patients who reported moderate and high physical activity were those who were motivated to be healthy. ${ }^{43}$ Lack of willpower, resources, and social support were the most frequently reported barriers in Oman. ${ }^{52}$

While this study utilised the barrier component of the EBBS questionnaire, these other studies used other instruments such as the 27-item Barriers to Being Active questionnaire. 
Table 1: Sociodemographic characteristics of the participants.

\begin{tabular}{|c|c|c|c|c|}
\hline Variables & $\begin{array}{l}\text { Frequency with } \\
\text { diabetes }\end{array}$ & Percentage (\%) & $\begin{array}{l}\text { Frequency without } \\
\text { diabetes }\end{array}$ & Percentage (\%) \\
\hline \multicolumn{5}{|l|}{ Age (years) } \\
\hline $25-34$ & 2 & 1.0 & 35 & 17.5 \\
\hline $35-44$ & 7 & 3.5 & 59 & 29.5 \\
\hline $45-54$ & 44 & 22.0 & 73 & 36.5 \\
\hline $55-64$ & 59 & 29.5 & 28 & 14.0 \\
\hline $65-74$ & 83 & 41.5 & 5 & 2.5 \\
\hline$\geq 75$ & 5 & 2.5 & 0 & 0.0 \\
\hline Total & 200 & 100 & 200 & 100.0 \\
\hline \multicolumn{5}{|l|}{ Sex } \\
\hline Male & 119 & 54.5 & 124 & 62.0 \\
\hline Female & 91 & 45.5 & 76 & 38.0 \\
\hline Total & 200 & 100.0 & 200 & 100.0 \\
\hline \multicolumn{5}{|l|}{ Marital status } \\
\hline Single & 13 & 6.5 & 30 & 15.0 \\
\hline Married & 101 & 50.5 & 132 & 66.0 \\
\hline Divorced & 8 & 4 & 3 & 1.5 \\
\hline Widowed & 57 & 28.5 & 25 & 12.5 \\
\hline Widower & 21 & 10.5 & 10 & 5 \\
\hline Total & 200 & 100.0 & 200 & 100.0 \\
\hline \multicolumn{5}{|l|}{ Occupation } \\
\hline Unemployed & 19 & 9.5 & 0 & 0.0 \\
\hline Civil servant & 46 & 23.0 & 165 & 82.5 \\
\hline Private & 12 & 6.0 & 0 & 0.0 \\
\hline Self employed & 35 & 17.5 & 35 & 17.5 \\
\hline Retired & 30 & 15.0 & 0 & 0.0 \\
\hline Farming & 27 & 13.5 & 0 & 0.0 \\
\hline Stay-at-home spouse & 31 & 15.5 & 0 & 0.0 \\
\hline Total & 200 & 100.0 & 200 & 100.0 \\
\hline \multicolumn{5}{|l|}{ Education } \\
\hline Informal & 35 & 17.5 & 0 & 0.0 \\
\hline Primary & 26 & 13.0 & 0 & 0.0 \\
\hline Junior secondary & 6 & 3.0 & 0 & 0.0 \\
\hline Senior secondary & 43 & 21.5 & 67 & 33.5 \\
\hline First degree & 80 & 40.0 & 101 & 50.5 \\
\hline Postgraduate & 10 & 5.0 & 32 & 16.0 \\
\hline
\end{tabular}


Table 2: Descriptive and comparative statistical analysis of physical activity levels among participants with and without diabetes.

\begin{tabular}{|l|l|l|l|l|l|l|}
\hline Physical activity & $\begin{array}{l}\text { Frequency of } \\
\text { people with } \\
\text { diabetes (\%) }\end{array}$ & $\begin{array}{l}\text { Frequency of } \\
\text { people without } \\
\text { diabetes (\%) }\end{array}$ & Total (\%) & t value & $\begin{array}{l}\text { Degrees of } \\
\text { freedom }\end{array}$ & value \\
\hline Low & $142(71.0)$ & $66(33.0)$ & $246(61.5)$ & 4.980 & 2 & \\
\hline Moderate & $47(23.5)$ & $104(52.0)$ & $113(28.3)$ & & \\
\hline High & $11(5.5)$ & $30(15.0)$ & $41(10.0)$ & \\
\hline Total & $200(100.0)$ & $200(100.0)$ & $400(100.0)$ & & \\
\hline
\end{tabular}

Table 3: Participants' mean and standard deviation scores for domains of barriers to exercise participation among diabetic and nondiabetic participants.

\begin{tabular}{|c|c|c|}
\hline Variables & $\begin{array}{l}\text { Diabetic } \\
\text { participants } \\
(\text { mean } \pm S D)\end{array}$ & $\begin{array}{l}\text { Nondiabetic } \\
\text { participants } \\
(\text { mean } \pm S D)\end{array}$ \\
\hline \multicolumn{3}{|l|}{ Exercise milieu subscale } \\
\hline 1. Places for me to exercise are too far away & $2.82 \pm 0.83$ & $3.20 \pm 0.81$ \\
\hline 2. I am too embarrassed to exercise & $2.98 \pm 0.78$ & $2.38 \pm 1.04$ \\
\hline 3. It costs too much to exercise & $2.90 \pm 0.79$ & $2.63 \pm 0.77$ \\
\hline 4. Exercise facilities do not have convenient schedule for me & $2.70 \pm 0.88$ & $2.74 \pm 0.66$ \\
\hline 5. There are too few places for me to exercise & $2.64 \pm 0.98$ & $2.90 \pm 0.95$ \\
\hline Mean & $2.81 \pm 0.85$ & $2.76 \pm 0.84$ \\
\hline \multicolumn{3}{|l|}{ Time expenditure subscale } \\
\hline 6. Exercise takes too much of my time & $2.68 \pm 0.92$ & $2.76 \pm 0.84$ \\
\hline 7. Exercise takes too much time from my family responsibility & $2.65 \pm 0.97$ & $2.68 \pm 0.81$ \\
\hline Mean & $2.66 \pm 0.94$ & $2.72 \pm 0.82$ \\
\hline \multicolumn{3}{|l|}{ Physical exertion subscale } \\
\hline 8. Exercise tires me & $2.87 \pm 0.86$ & $2.64 \pm 0.78$ \\
\hline 9. I am fatigued by exercise & $2.94 \pm 0.84$ & $3.00 \pm 0.85$ \\
\hline Mean & $2.90 \pm 0.85$ & $2.82 \pm 1.63$ \\
\hline \multicolumn{3}{|l|}{ Family discouragement subscale } \\
\hline 10. My significant other(s) does not encourage exercising & $2.65 \pm 0.96$ & $2.70 \pm 0.69$ \\
\hline 11. My family members does not encourage me to exercise & $2.77 \pm 0.93$ & $2.66 \pm 0.90$ \\
\hline Mean & $2.71 \pm 0.95$ & $2.68 \pm 0.80$ \\
\hline Total (mean $\pm S D)$ & $2.78 \pm 0.88$ & $2.75 \pm 0.83$ \\
\hline
\end{tabular}

SD: standard deviation. 
Time expenditure and family discouragement were the least reported barriers to exercise in participants with and without diabetes, respectively. The cost of exercising, being too embarrassed to exercise, and distance to exercise facilities were highly rated as important barriers to physical activity by respondents, even when they had time or were encouraged by their families. In agreement with this study's findings, family discouragement and busy work schedules (time) were important barriers to being physically active in Nepal; ${ }^{27}$ the United Arab Emirates,53 Spain; ${ }^{54}$ Denmark; ${ }^{55}$ and North Carolina, USA. ${ }^{45}$ Health conditions, pain, environment, lack of accessibility, and time appear to be potential barriers to physical activity among older adult populations. ${ }^{56,57}$ The presence of these barriers could have accounted for the low levels of physical activity reported in this study.

\section{CONCLUSIONS}

This study made information available on the levels and perceived barriers of physical activity among patients with diabetes from Nigeria. There is a need to make observations in a similar population consisting of people with or without diabetes in order to detect and understand outcomes, so as to use the data from nondiabetic patients to effectively manage diabetes and the data from patients with diabetes to effectively delay disease onset. This study revealed that the majority of patients with diabetes had low physical activity level while nondiabetic participants had moderate physical activity level. However, physical exertion was reported by both patients with diabetes and nondiabetic participants as the greatest barrier to exercise participation; time expenditure and family discouragement were, respectively, the least reported barrier to exercise among participants. Recommendations are therefore that physiotherapy should be made a compulsory treatment protocols for patients with diabetes in tertiary institutions. Public awareness on the health benefits of exercise in prevention and management of diabetes should be implemented by the federal ministry of health. The federal government of Nigeria, in collaboration with state and local governments, should provide strategically placed exercise facilities for people that are willing to exercise. Physiotherapists and other health practitioners who treat patients with diabetes should always assess their physical activity level and perceived barriers to exercise. Further research involving more subjects in a very large population should be studied.

\section{Limitations}

The participants in this study were individuals visiting the clinic at the time of data collection and were not representative of all the patients with diabetes in the area. Therefore, there is limited generalisability of the findings. The crosssectional design of this study does not allow for cause and effect inference.

\section{References}

1. World Health Organization (WHO). Non-communicable disease country profiles. 2018. Available at: https:// www.who.int/nmh/publications/ncdprofiles-2018/en/. Last accessed: 4 January 2021.

2. Forouzanfar MH et al. Global, regional, and national comparative risk assessment of 79 behavioural, environmental and occupational, and metabolic risks or clusters of risks, 1990-2015: a systematic analysis for the global burden of disease study. Lancet. 2016;388(10053):1659-724.

3. Farzadfar $\mathrm{F}$ et al. Effectiveness of diabetes and hypertension management by rural primary healthcare workers (Behvarz workers) in Iran: a nationally representative observational study. Lancet. 2012;379(9810):47-54
4. Ganu D et al. Physical disability and functional impairment resulting from Type 2 diabetes in sub-Saharan Africa: a systematic review. Afr J Diabetes Med. 2016;24(1):10-4.

5. Pastakia SD et al. Diabetes in subSaharan Africa- from policy to practice to progress: targeting the existing gaps for future care for diabetes. Diabetes Metab Syndr Obes. 2017;10:247-63.

6. Fattahi A et al. Physical activity and its related factors among Type 2 Diabetic patients in Hamadan. Iran J Diabetes Obes. 2014;6(2):85-92.

7. World Health Organization (WHO). Definition, diagnosis and classification of diabetes mellitus and its complications, Part 1. 1999. Available at: https://apps.who.int/iris/ handle/10665/66040. Last accessed: 4 January 2021.

8. International Diabetes Federation (IDF). IDF Diabetes Atlas. 9th edition. 2019. Available at: http://www.idf. org/diabetesatlas. Last accessed: 4 January 2021.

9. Hall $V$ et al. Diabetes in Sub-Saharan Africa 1999-2011: epidemiology and public health implications. A systematic review. BMC Public Health. 2011;11(1):11-564.

10. Non-Communicable Disease Risk Factor Collaboration (NCD-RisC). Worldwide trends in diabetes since 1980: a pooled analysis of 751 population-based studies with 4.4 million participants. Lancet. 2016;387(10027):1513-30. 
11. Zhou B et al. Worldwide trends in diabetes since 1980: a pooled analysis of 751 population-based studies with 4.4 million participants. Lancet. 2016;387(10027):1513-30.

12. World Health Organization (WHO). Global report on diabetes. 2016. Available at: http://apps.who.int/iris/ bitstream/10665/204871/1/97892415 65257 eng.pdf Last accessed: 4 January 2021.

13. Jaffar S, Gill G. The crisis of diabetes in Sub-Saharan Africa. Lancet Diabetes Endocrinol. 2017;5(8):574-5.

14. Akinkugbe OO. Non-communicable diseases in Nigeria: national survey (final report) on hypertension, coronary heart disease, diabetes mellitus, haemoglobinopathies, G6PD deficiency and anaemia. National Expert Committee on Noncommunicable Diseases. Federal Ministry of Health and Social Services. Lagos, Nigeria, 1997. Available at: https://scholar.google.com/ scholar?hl=en\&q=+Non+ communicable+diseases+in+Nigeria:+ national+survey+(final+report)+on+ hypertension+coronary+heart+ disease+diabetes+mellitus+G6PD+ deficiency+and+anaemia. Last accessed: 4 January 2021.

15. Christensen DL et al. Prevalence of glucose intolerance and associated risk factors in rural and urban populations of different ethnic groups in Kenya. Diab Res Clin Prac. 2009;84(3):303-10.

16. Ahren B, Corrigan CB. Prevalence of diabetes mellitus in north-western Tanzania. Diabetologia. 1984;26:3336.

17. Uloko AE et al. Prevalence and risk factors for diabetes mellitus in Nigeria: a systematic review and meta-analysis. Diabetes Ther. 2018:9(3):1307-16.

18. World Bank. World development indicators. 2016. Available at: https:// datacatalog.worldbank.org/dataset/ world-development-indicators. Last accessed: 5 January 2021.

19. Bailey SL et al. Diabetes mellitus in Zambia and the western cape province of South Africa: prevalence, risk factors, diagnosis and management. Diabetes Res Clin Pract. 2016;118:1-11.

20. Ley $\mathrm{SH}$ et al. Prevention and management of Type 2 diabetes: dietary components and nutritional strategies. Lancet. 2014;383(9933):1999-2007

21. Bakari A, Onyemelukwe G. Insulin resistance in Type 2 diabetic Nigerians. Int J Diabetes Metab. 2005:13:24-7.

22. Kibirige $D$ et al. Understanding the manifestation of diabetes in SubSaharan Africa to inform therapeutic approaches and preventive strategies: a narrative review. Clin Diabetes
Endocrinol. 2019;5(2):DOI:10.1186/ s40842-019-0077-8.

23. Mohamed S et al. Prevalence and factors associated with pre-diabetes and diabetes mellitus in Kenya: results from a national survey. BMC Public Health. 2018;18(3):1215.

24. Adeleke OR, Ayenigbara GO. Preventing diabetes mellitus in Nigeria: effect of physical exercise, appropriate diet, and lifestyle modification. Int J Diabetes Metab. 2019;25:3-4.

25. Agofure $\mathrm{O}$ et al. Knowledge of dietary and medical management of Type-2 diabetes in an urban and rural community of Delta State Nigeria. Afr J Diabetes Med. 2018;26(1):12-5.

26. Odenigbo MA, Inya-Osuu J. Knowledge, attitudes and practices of people with Type-2 diabetes mellitus in a tertiary health care centre, Umuahia, Nigeria. J Diabetes Metab, 2012;3:187-91.

27. Kadariya S, Aro AR. Barriers and facilitators to physical activity among urban residents with diabetes in Nepal. PLoS ONE. 2018;13(6):e0199329.

28. Kennerly AM, Kirk A. Physical activity and sedentary behaviour of adults with Type 2 diabetes: a systematic review. Pract Diabetes. 2018;35(3):869.

29. World Health Organization (WHO). Global strategy on diet, physical activity and health. 2018. Available at: http://www.who.int/ dietphysicalactivity/pa/en/. Last accessed: 5 January 2021.

30. American Diabetes Association (ADA). Standards of medical care in diabetes - 2016. Diabetes Care. 2016;39(Suppl 1):s27.

31. Shields $\mathrm{N}$ et al. Perceived barriers and facilitators to physical activity for children with disability: a systematic review. Br J Sports Med. 2012;46(14):989-97.

32. Mwaura LW et al. Effect of distance on access to health services among women with Type 2 diabetes in a rura community in Kenya. Afr J Diabetes Med. 2017;25(1):18-20.

33. Achigbu EO et al. Knowledge and impact of diabetes in patients in a tertiary clinic in southeast Nigeria. Afr J Diabetes Med. 2015;23:15 7. Available at: https://www. africanjournalofdiabetesmedicine. com/articles/8.\%20AJDM-497\%20 (Achigbu).pdf. Last accessed: 5 January 2021.

34. World Health Organization (WHO). Diabetes country profiles. 2016. Available at: https://who.int/diabetes/ country-profiles/nga_en.pdf?ua=1 Last accessed: 5 January 2021.

35. Idowu OA et al. Perceived barriers to physical activity among
Nigerian stroke survivors. Pan Afr Med. 2015;21:274;DOI:10.11604/ pamj.2015.21.274.6669.

36. Nolan R et al. Self-reported physical activity using the international physical activity questionnaire (IPAQ) in Australian adults with Type 2 diabetes, with and without peripheral neuropathy. Can J Diabetes. 2016;40(6):576-9.

37. American College of Sports Medicine, Guidelines for graded exercise testing and exercise prescription (1980) 2nd edition, Philadelphia: Lea \& Febiger.

38. Sechrist KR et al. Development and psychometric evaluation of the exercise benefits/barriers scale. Res Nurs Health. 1987;10(6):357-65.

39. Oyewole $\mathrm{OO}$ et al. Physical activity among Type 2 diabetic adult Nigerians. Ann Afr Med. 2014;13(4):189-94.

40. Kabanda AM, Phillips JS. Physical activity among adults with diabetes mellitus in Rwanda. Sahara J. 2011;17(2):239-47.

41. Gatimu SM et al. Prevalence and determinants of diabetes among older adults in Ghana. BMC Public Health. 2016;16:1174.

42. Shiriyedeve $\mathrm{S}$ et al. Factors associated with physical activity in Type 2 diabetes mellitus patients at a public clinic in Gaborone, Botswana, in 2017. Afr J Prim Health Care Fam Med. 2019;11(1):a2036.

43. Shazwani $\mathrm{N}$ et al. Assessment of physical activity level among individuals with Type 2 diabetes mellitus at Cheras Health Clinic, Kuala Lumpur. Malays J Nutr. 2010;16(1):10112.

44. Ranasinghe DC et al. Evaluation of physical activity among adults with diabetes mellitus from Sri Lanka. Int Arch Med. 2014;7:15.

45. Donahue KE et al. Identifying supports and barriers to physical activity in patients at risk for diabetes. Prev Chronic Dis. 2006;3(4):A119.

46. Morrato EH et al. Physical activity in U.S. adults with diabetes and at risk for developing diabetes. Diabetes Care. 2007;30(2):203-9.

47. Kriska AM et al. Physical activity, obesity and the incidence of Type 2 diabetes in a high-risk population. Am J Epidemiol. 2003;158(7):669-75.

48. Craig $C L$ et al. International physical activity questionnaire: 12-country reliability and validity. Med Sci Sports Exerc. 2003;35(8):1381-95.

49. World Health Organization (WHO). "Move for health": world health day. 2002. Available at: https://www.who. int/docstore/world-health-day/2002/ euro_factsheet.pdf. Last accessed: 5 January 2021. 
50. World Health Organization (WHO) Non-communicable disease prevention and health promotion. 2003. Available. https://www.who.int/ mip/2003/progress/en/nmhmip2003. pdf. Last accessed: 5 January 2021.

51. Dalibalta S, Davison G. Perceived exercise benefits and barriers of a mixed student population in the United Arab Emirates. Int J Sci Res Innov Tech. 2016;3(2):115-39.

52. Alghafri $T$ et al. Perceived barriers to leisure time physical activity in adults with Type 2 diabetes attending primary healthcare in Oman: a cross-sectional survey. BMJ Open. 2017;7(11):e016946.

53. Al-Kaabi $J$ et al. Physical activity and reported barriers to activity among Type 2 diabetic patients in the United Arab Emirates. Rev Diabet Stud. 2009;6(4):271-8

Nicolas Lopez J et al. Barriers to physical activity in people with diabetes residing in Spain. Atena $J$ Public Health. 2020;2:3.
54. Lidegaard LP et al. Barriers to and motivators for physical activity among people with Type 2 diabetes: patients' perspectives. Diabet Med. 2016;33(12):1677-85

55. Schuler PB et al. Barriers and motivations to exercise in older African American and European American women. Calif J Health Promot. 2006;4(3):128-34.

56. Schutzer KA, Graves BS. Barriers and motivations to exercise in older adults. Prev Med. 2004:39(5):1056-61. 